\title{
Multiwavelength view of SDSS galaxies
}

\author{
M. Obrićc $\dot{1}^{1,2}$, Ž. Ivezić ${ }^{1,3}$, R. H. Lupton ${ }^{1}$, G. Kauffmann ${ }^{4}$, G. R. \\ Knapp ${ }^{1}$, J. E. Gunn ${ }^{1}$, D. Schlegel ${ }^{1}$, M. A. Strauss ${ }^{1}$ and S. Anderson ${ }^{3}$ \\ ${ }^{1}$ Princeton University, Princeton, USA \\ ${ }^{2}$ Kapteyn Institute, Groningen, The Netherlands \\ ${ }^{3}$ University of Washington, Seattle, USA \\ ${ }^{4}$ Max-Planck-Inst. für Astroph., Garching, Germany
}

\begin{abstract}
We summarize the detection rates at wavelengths other than optical for $\sim 99,000$ galaxies from the Sloan Digital Sky Survey (SDSS) Data Release 1 "main" spectroscopic sample. The analysis is based on positional cross-correlation with source catalogs from ROSAT, 2MASS, IRAS, GB6, FIRST, NVSS and WENSS surveys. We find that the rest-frame UV-IR broad-band galaxy SEDs form a remarkably uniform, nearly one parameter, family. As an example, the SDSS $u$ and $r$ band data, supplemented with redshift, can be used to predict $K$ band magnitudes measured by 2MASS with an rms scatter of only 0.2 mag; when measurement uncertainties are taken into account, the astrophysical scatter appears not larger than $\sim 0.1$ mag.
\end{abstract}

\section{The fractions of SDSS galaxies detected at other wavelengths}

We report initial results from a program aimed at the characterization of multiwavelength properties of galaxies detected by SDSS. The details about the positional crosscorrelation method, and analysis of matched samples, will be described elsewhere (Obrić et al. 2004); here we summarize the detection rates and demonstrate a tight correlation between the UV and IR colors of galaxies.

Table 1 lists the detection rates for galaxies from SDSS "main" spectroscopic sample (a flux-limited sample, $r_{P e t}<17.77$, for details see Strauss et al. 2002) by ROSAT, 2MASS, IRAS, GB6, FIRST, NVSS and WENSS surveys. Galaxies are separated into those with and without emission lines (using line measurements and classification criteria from Kauffmann et al. 2003), and emission line galaxies are further separated, using Baldwin-Phillips-Terlevich diagram, into "star-forming", "AGNs", and "unclassified". The detection rate of SDSS galaxies is below $10 \%$ in all surveys except 2MASS.

\section{Predicting 2MASS K flux from UV/optical SDSS flux}

Galaxies form a nearly one-dimensional sequence in various optical color-color diagrams (the correlation among colors is especially tight for rest-frame colors, with a scatter of only $\sim 0.03 \mathrm{mag}$ perpendicular to the locus, Smolčić et al. 2004). We report here that this one-dimensionality of galaxy spectral energy distributions (SEDs) extends to 2MASS wavelengths: it is possible to predict 2MASS $K$ band flux with an rms of 0.2 mag using SDSS $u$ and $r$ band measurements $\dagger$.

The SDSS-based $K$ magnitude prediction is determined from $K_{S D S S}=r_{P e t}-(r-K)$, where $r_{P e t}$ is the SDSS $r$ band Petrosian magnitude (Strauss et al. 2002), and $(r-K$ ) is a best fit to the observed $r-K$ colors for SDSS/2MASS galaxies. It depends on $u-r$ color (essentially a position along the Hubble sequence), and on redshift, to account

$\dagger$ We use UV/visual fluxes ( $u$ and $r$ bands) to predict near-IR flux because this is the "hardest" wavelength combination with most astrophysical implications (according to "common wisdom", such a relationship should not be very accurate due to the effects of starbursts and dust extinction); predicting, for example, 2MASS $J$ band flux from SDSS $z$ band flux is trivial because these two bands are adjacent in wavelength space. 


\begin{tabular}{cccccccc}
\hline \multicolumn{1}{c}{ Table 1. The detection rates of SDSS galaxies at other } & wavelengths \\
Catalog & Wavelength & Total & No emission & Emission & AGN & SF & Unclassified \\
$\Sigma_{S D S S}\left(\# / \mathrm{deg}^{2}\right)$ & UV-IR & 72.86 & 39.38 & 33.48 & 11.81 & 7.54 & 13.44 \\
2MASS XSC [\%] & near-IR & 38.06 & 35.72 & 39.64 & 63.79 & 10.69 & 34.00 \\
IRAS [\%] & far-IR & 2.25 & 0.29 & 3.73 & 3.73 & 2.31 & 4.50 \\
GB6 [\%] & $6 \mathrm{~cm}$ & 0.37 & 0.44 & 0.26 & 0.43 & 0.17 & 0.16 \\
FIRST [\%] & $20 \mathrm{~cm}$ & 3.86 & 2.76 & 4.68 & 8.06 & 0.98 & 3.40 \\
NVSS [\%] & $20 \mathrm{~cm}$ & 7.78 & 7.06 & 7.85 & 9.54 & 5.68 & 7.50 \\
WENSS [\%] & $92 \mathrm{~cm}$ & 0.61 & 0.77 & 0.40 & 0.61 & 0.25 & 0.30 \\
ROSAT [\%] & X-ray & 0.27 & 0.27 & 0.20 & 0.37 & 0.10 & 0.098 \\
\hline
\end{tabular}
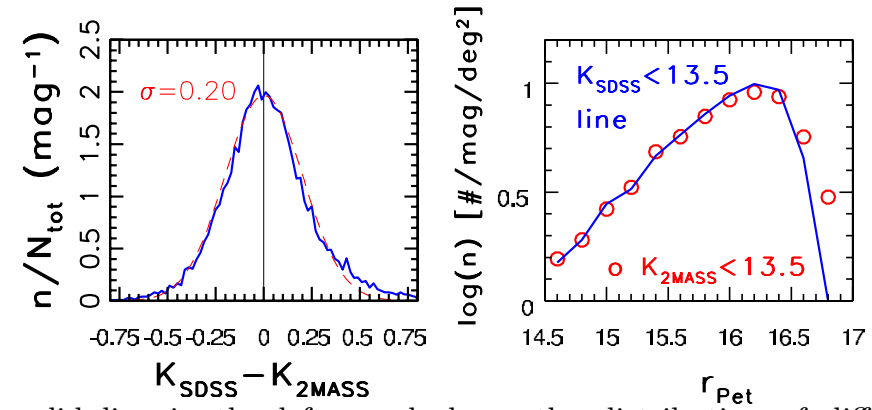

Figure 1. The solid line in the left panel shows the distribution of differences between SDSS-predicted and 2MASS-measured $K$ band flux. The dashed line is a Gaussian with $\sigma=0.20$ mag. The right panel shows the $r$ band counts of SDSS-predicted (line) and 2MASS-measured (symbols) galaxies with $K<13.5$ (2MASS XSC completeness limit).

for K-correction effects (we use default 2MASS magnitudes and SDSS model colors):

$$
(r-K)=A+B(u-r)+C(u-r)^{2}+D(u-r)^{3}+E z+F z^{2}
$$

where $(\mathrm{A}, \mathrm{B}, \mathrm{C}, \mathrm{D}, \mathrm{E}, \mathrm{F})=(1.115,0.940,-0.165,0.00851,4.92,-9.10)$, and $z$ is redshift. This fit predicts 2MASS $K$ band magnitudes with an rms scatter of 0.20 mag, which depends on neither color nor redshift, and is nearly Gaussian (see Figure 1).

To correct for aperture and resolution effects between SDSS and 2MASS, that presumably depend on galaxy profile, or nearly equivalently on galaxy color (Strateva et al. 2000), we add to $(r-K)\left(0.496-0.154 R_{50}^{z}\right)$ for galaxies with $u-r<2.22$ and $\left(0.107-0.045 R_{50}^{z}\right)$ for redder galaxies, where $R_{50}^{z}$ is the radius containing $50 \%$ of Petrosian flux in the $z$ band. This correction has a negligible effect on rms scatter, and only removes a correlation of $K_{S D S S}-K_{2 M A S S}$ residuals with galaxy size.

The median residuals, as a function of $u-r$ and $z$, do not exceed 0.03 mag. The rms scatter decreases to $0.15 \mathrm{mag}$ at the bright end $(K<12)$. Given typical measurement errors in $u, r, R_{50}^{z}$ and $K$, we conservatively conclude that the true astrophysical scatter of $K$ band magnitudes predicted from the blue part of SED is less than $\sim 0.1$ mag. Similarly, the relation $(J-K)=2.172 z+0.966$, where $z$ is redshift, predicts $J-K$ measured by 2MASS with an rms scatter of $0.11 \mathrm{mag}(0.07 \mathrm{mag}$ at the bright end), and no significant residuals with respect to $K, u-r$ and redshift. These tight correlations demonstrate remarkable one-dimensionality of galaxy spectral energy distributions from UV to IR wavelengths.

\section{References}

Kauffmann, G., et al. 2003, MNRAS, 341, 33

Obrić, M., et al. 2004, MNRAS, in prep.

Smolčić, V., et al. 2004, MNRAS, in prep.

Strateva, I., et al. 2001, AJ, 122, 1861

Strauss, M. A., et al. 2002, AJ, 124, 1810 